\section{Ein Stück weiter im Umweltschutz}

\author{
Ende dieses Jahres wird eine überarbeitete Fassung der ISO 14001 Norm in \\ Kraft treten. Der Text der Norm liegt jetzt schon vor. Was auf Anwender durch \\ die zahlreichen Änderungen der Norm zukommt, wird im Folgenden verdeutlicht \\ und analysiert.
}

$\mathrm{D}$ Von Jan Uwe Lieback entstand aus der Neufassung der ISO 9001:2000. Sukzessive sollen die Normen angeglichen werden. Dem trug bereits die ISO 9001:2000 Rechnung. Zugleich sollten die Begriffsdefinitionen dieses Normenpaars vereinheitlicht werden. Bereits abgeschlossen ist zwischenzeitlich die gelungene Vereinheitlichung der Auditierungsnormen zur ISO 19001. Von Anfang an war geplant, mit der Revision der ISO 14001 keine neuen Anforderungen zu schaffen. Es war zwar deutlich geworden, dass einige Formulierungen zu Interpretationsschwierigkeiten führten. Diese sollten deshalb mit einer Neufassung vermieden werden. Durch eine Erhöhung der Verständlichkeit sollten ferner der Einstieg für kleine und mittlere Unternehmen (KMU) erleichtert werden

Zum vorliegenden Entwurf lief die Frist Änderungsvorschläge einzubringen Ende Januar 2004 aus. Es wird erwartet, dass die Neufassung im Oktober 2004 ohne Übergangsregelung Gültigkeit erlangt. Folgend werden die wesentlichsten Veränderungen näher beleuchtet.

\section{- Umweltpolitik}

In der neuen Fassung der ISO 14001 wird hervorgehoben, dass die Umweltpolitik nicht nur ein wohlfeiles Schriftstïck der Selbstdarstellung ist. Vielmehr soll sie erkennbar auf dem definierten Umfang des Umweltmanagementsystems (UMS) aufbauen und dabei den strategischen Ansatz des Unternehmens auf der Basis der signifikanten Umweltaspekte aufgreifen, die im Vorfeld der Aufstellung der Politik zu ermitteln sind. Hervorgehoben wird auch, dass sich dessen spezifische Eigenheiten, der Umfang und die Umweltauswirkungen der Aktivitäten, Produkte und Dienstleistungen im Inhalt widerspiegeln sollen.

Umweltpolitik soll jetzt nicht nur ,,bekannt gemacht", wie es in der alten Fassung heißt, sondern „allen Personen gegenüber kommuniziert" werden, die für die Organisation oder im Interesse der

Zur besseren Übersicht sollen, auch in Analogie an EMAS, Kriterien und Methoden für die Auswahl der signifikanten Aspekte festgelegt werden. Das sind die, welche die Umweltauswirkungen des Anwenders maßgeblich beeinflussen und deren Verringerung die Umweltleistung verbessern kann. Ergebnisse sind zu dokumentieren und regelmäßig zu aktualisieren. Signifikante Umweltaspekte sollen bei der Entwicklung,

Einen Überblick über alle bisher erschienenen Hefte des Informationsdienstes "Ökologisches Wirtschaften" finden Sie auf unseren Internetseiten

\section{http://www.oekom.de}

Wir freuen uns auf Ihren Besuch! der Einführung und der Aufrechterhaltung des Umweltmanagements besonders berücksichtigt werden. Verfahren, Prozesse und Anweisungen des UMS sollen sich vorwiegend mit diesen signifikanten Aspekten auseinandersetzen.

\section{Einbeziehung umweltrechtlicher Forderungen}

Punkt 4.3.2 des Entwurfs räumt mit irrigen Vorstellungen auf. So gab die deutsche Übersetzung der Norm bisher Anlass zur Vermutung, dass nur die von einer Organisation als bedeutsam angesehenen umweltrechtlichen Vorschriften zu berücksichtigen sind. Mit dieser Auslegung macht der neue Text Schluss. Eindeutig stellt er heraus, dass alle anwendbaren Gesetze zu berïcksichtigen sind, die Umweltaspekte des ISO 14001 Anwenders betreffen. Er hat festzulegen, wie diese Anforderungen seine Aspekte berïhren. Dies ist sogar in einer aktuell zu haltenden Verfahrensanweisung festzulegen.

An einigen Stellen gibt die Norm Forderungen nach festgelegten Verfahren auf und beschränkt sich auf die Aufzeichnung bestimmter Informationen; hier verlangt sie ein solches explizit.

Zudem fordert der einzige neue Punkt 4.5.2 „Prïfung der Einhaltung der Gesetze", dass jede Organisation ebenfalls ein Verfahren benötigt, um periodisch die Übereinstimmung mit den anwendbaren Gesetzen und Vorschriften zu prüfen. Nur so kann der Anwender seine Verpflichtung einlösen, die er in der Umweltpolitik abgeben musste. unter der Adresse:
Die Abschnitte zu Zielen und Programmen wurden zusammengefasst. Letztlich setzt sich jedes Umweltprogramm aus verschiedenen Einzelzielen zusammen. Im Programm wird erläutert, wie sie konkret umgesetzt werden sollen. Man darf auf die Übersetzung des deutschen Textes gespannt sein, ob er mit dem Verwirrspiel um Ziele und Einzelziele aufräumt. Der englische Originaltext ist mit den Worten „objectives“ und ,targets“ verständlicher als der deutsche. Objectives sind Richtungsziele, die sich aus der Strategie eines Anwenders beziehungsweise der Erkenntnis seiner signifikanten Umweltaspekte ergeben. Diese sind auf den einzelnen Ebenen einer Organisation auf konkrete Ziele herunter zu brechen.

Der neue Text macht immerhin mit der Vorstellung Schluss, Umweltziele müssten nur bis zum nächsten internen oder externen Audit gesetzt werden. Im Anhang wird darauf verwiesen, dass

\section{Umweltziele und Umweltprogramme}


bei der Zielfestlegung sowohl kurzfristige als auch langfristige Probleme berïcksichtigt werden sollen. Somit können Ziele über einen Zeitraum gespannt und mit Maßnahmen untersetzt werden, die in Jahresscheiben abzuarbeiten sind.

\section{Kommunikation}

Hat ein Anwender entschieden, über seine signifikanten Umweltaspekte zu kommunizieren, kann er das nicht nach Gutdünken tun. Will er kommunizieren, muss er dafür Methoden und Verfahren festsetzen und eine gleich bleibende Kommunikation über gute oder schlechte Nachrichten sicherstellen.

Hinsichtlich der Einbeziehung von Mitarbeitern wird nicht mehr von ,Mitarbeitern“, sondern „Personen“ gesprochen, die Aufgaben im Auftrag ausführen, welche das Potenzial haben, signifikante Umweltauswirkungen hervorzurufen. Der Anwender des Zertifizierungsverfahrens muss sicherstellen, dass jede dieser „Personen“ ausgebildet, trainiert und erfahren ist. Er hat Sorge dafür zu tragen, dass die Betreffenden gegebenenfalls eine Unterweisung oder ein Training erhalten.

Im Anhang wird darauf verwiesen, dass Anwender von ,in ihrem Einwirkungsbereich“ tätigen Fremdfirmen fordern sollen, die erforderliche Kompetenz und notwendige Schulung ihrer Mitarbeiter nachzuweisen. Nur so kann schließlich sichergestellt werden, dass im Einflussbereich des UMS wirklich alle gesetzlichen Regelungen angewendet werden. Bei den Punkten Dokumentationen und Aufzeichnungen gibt es wenig Neues. Beide Begriffe werden verdeutlicht und jetzt wie in der ISO 9001:2000 verwendet. Insbesondere wird darauf verwiesen, wo nötig, Aufzeichnungen zum Nachweis der Systemfunktionen zu führen. Korrektur- und Vorbeugemaßnahmen werden in der Neufassung konkretisiert. Nach Identifikation von Abweichungen beziehungsweise des Risikos von Abweichungen sollen für deren Minimierung Maßnahmen ergriffen werden. Ursachen sollen beseitigt, mindestens aber die Auswirkungen begrenzt werden. Dazu sind Maßnahmen festzulegen, deren Umsetzung überwacht und dokumentiert wird.

\section{Audit und Review}

Durch Einschub der Rechtsprüfung rückt der Punkt Audits um einen Zähler auf Punkt 4.5.5 nach hinten. Änderungen der Anforderungen gibt es nicht. Findet eine Compliance Prüfung parallel zur Durchführung des internen Audits statt, müssen Ziel und Umfang dieses Auditteils definiert sein und das Ergebnis im Bericht hervorgehoben werden.
Ein Segen sind die Klarstellungen zur Durchführung des Reviews. Es wird aus seiner Rolle als Bericht, den ein Beauftragter oft nur von der Geschäftsleitung gegenzeichnen lässt, endgültig in einen aktiven Prozess überführt.

Bereits unter Punkt 4.1 wird von Neuanwendern gefordert, vor jeder weiteren Handlung erst einen Review auf Basis der identifizierten Umweltaspekte sowie der rechtlichen Umweltforderungen durchzuführen. Darin soll der Umfang des Systems festlegt werden, auf dem die Politik aufbauen soll. Für die regelmäßigen Reviews setzt die neue Formulierung klare Vorgaben. Neben anderen Kriterien werden mögliche Verbesserungen des UMS, der Politik, der strategischen Ziele sowie der Einzelziele gefordert.

Dieser aktive Charakter wird zum Beispiel bei der Beschreibung der Informationsbasis deutlich, auf die sich das Review stïtzen soll. Neben den Ergebnissen interner Audits als Erkenntnisquelle werden die Ergebnisse der Kommunikation mit interessierten Kreisen, die Leistungen des UMS anhand von Zahlen, der Grad der Zielerreichung, der Status der Korrektur- und Vorbeugemaßnahmen sowie der Stand der Umsetzung der Beschlüsse und Ergebnisse des letzten Reviews genannt. Ausdrücklich wird gefordert, im Review den gesamten Umfang des UMS periodisch zu berücksichtigen, unter Umständen über Teilreviews in einem längeren Zeitraum.

\section{- Fazit}

Im Ergebnis erreicht der neue Text der ISO 14001 die gesteckten Zielen. Interessant wird, ob aus der deutschen Übersetzung die strategische Verbindung der signifikanten Umweltaspekte mit den daraus abzuleitenden Richtungszielen als Basis für die Einzelziele aller Bereiche erkennbar wird. Sicher ist, dass die systematische Verbindung dieser Aufgaben eine der wesentlichsten Arbeitsfelder der Berater, Auditoren und Anwender sein wird.

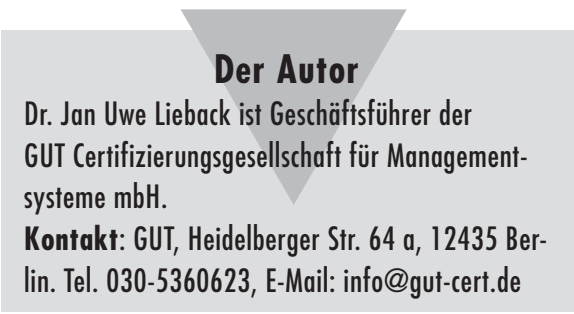

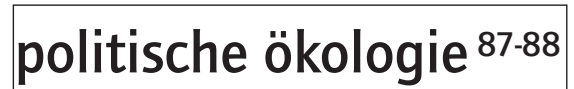

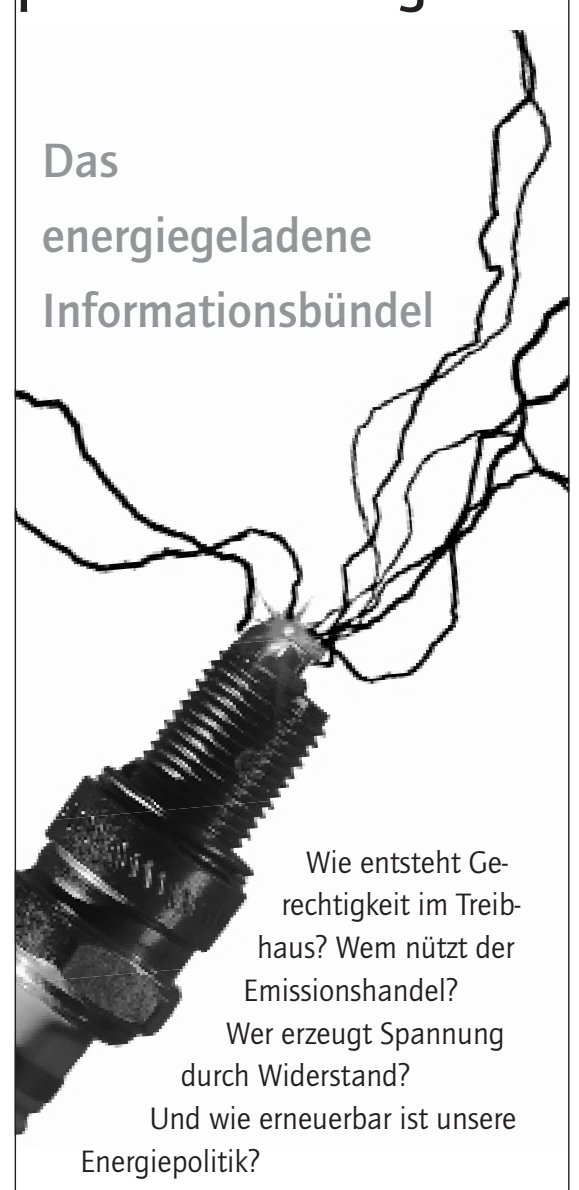

Lesen Sie, worüber vor und hinter den Kulissen der Energie- und Klimapolitik diskutiert wird.

Mit Beiträgen von H. Breyer, H. Graßl, C. Bals, M. Jänicke, F. Matthes, H. E. Ott, K. Pachauri, H. Scheer u.v.m.

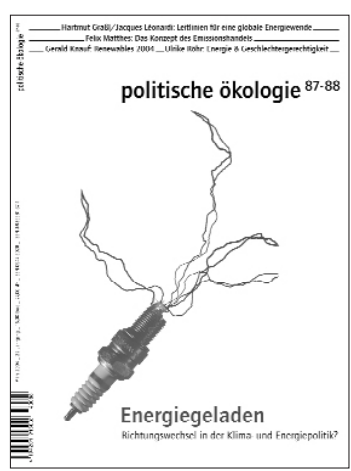

Stillen Sie Ihre Neugier!
_Fordern Sie Heft 87-88 an
_15,- $€$ (inkl. Versand)
_ISBN 3-936581-37-1
_ökom verlag
_Fax 089/544 18449
_www.oekom.de

neugier@oekom.de 
(c) 20I0 Authors; licensee IÖW and oekom verlag. This is an article distributed under the terms of the Creative Commons Attribution Non-Commercial No Derivates License (http://creativecommons.org/licenses/by-nc-nd/3.o/), which permits unrestricted use, distribution, and reproduction in any medium, provided the original work is properly cited. 